\title{
Fast beam steering and agile wavefront control with an optical phased array
}

Gozzard, David, Spollard, James, Roberts, Lyle, Sibley, Paul, McClelland, David, et al.

David R. Gozzard, James T. Spollard, Lyle E. Roberts, Paul G. Sibley, David E. McClelland, Daniel A. Shaddock, "Fast beam steering and agile wavefront control with an optical phased array," Proc. SPIE 11200, AOS Australian Conference on Optical Fibre Technology (ACOFT) and Australian Conference on Optics, Lasers, and Spectroscopy (ACOLS) 2019, 1120010 (30 December 2019); doi: 10.1117/12.2539892

SPIE. Event: ANZCOP, 2019, Melbourne, Australia 


\title{
Fast beam steering and agile wavefront control with an optical phased array
}

\author{
David R. Gozzard ${ }^{\mathrm{a}}$, James T. Spollard ${ }^{\mathrm{a}}$, Lyle E. Roberts ${ }^{\mathrm{a}}$, Paul G. Sibley ${ }^{\mathrm{a}}$, David E. \\ McClelland $^{\mathrm{a}}$, and Daniel A. Shaddock ${ }^{\mathrm{a}}$ \\ aResearch School of Physics and Engineering, The Australian National University, Canberra, \\ ACT, Australia
}

\begin{abstract}
Optical phased arrays (OPAs) are a solid-state device able to manipulate the distribution of optical power without the use of mechanical beam steering systems and have potential applications in free-space laser communications, target acquisition and tracking, and interferometry. Here we present a scalable OPA and digital control architecture capable of steering a laser beam at $\mathrm{MHz}$ frequencies, and having arbitrary control over the beam wavefront.
\end{abstract}

Keywords: optical phased array, field-programmable gate array, fats steering, wavefront control, phase sensing

\section{INTRODUCTION}

Laser beam steering systems are crucial to technologies such as light detection and ranging (LIDAR), remote sensing, and communications. Mechanical beam steering technologies are often limited in their steering bandwidth and scan patterns due to inertia of the components. These systems are also subject to mechanical wear which makes them a high-risk option for applications such as onboard spacecraft. Solid-state OPAs are not limited by inertia or mechanical wear, making them an attractive alternative for systems that require robust and agile beam steering. OPAs work by interfering multiple separate emitters to form a coherent and contiguous optical wavefront. By controlling the phase of each emitter in the array, it is possible to steer the beam in the far-field. ${ }^{1}$ In this talk we present the design of a scalable digitally controlled OPA. We describe the experimental demonstration of the phase sensing and control system, and progress towards fast ( $\mathrm{MHz}$ ) steering speeds.

\section{METHODS AND TECHNIQUE}

The OPA presented here uses EOMs to continuously control the phase of each element in the array, enabling arbitrary control of the wavefront and $\mathrm{MHz}$ bandwidth beam steering. Phase sensing and control is performed on a field-programmable gate array (FPGA). The phases of individual emitters are measured using digitally enhanced heterodyne interferometry (DEHI) ${ }^{2}$ which uses pseudo-random bit sequences (PRBS) encoded onto each emitter channel to distinguish and measure the phase of that emitter.

Light from a laser is split and guided through EOMs which apply a PRBS code, steering signal, and phase synchronization feedback to each channel, and launched into free-space (Fig. 1). A local oscillator channel is frequency shifted using an acousto-optic modulator for heterodyne detection at the photodetector. In the FPGA, the PRBS codes are demodulated and the signals are sent to separate phasemeters that measure the phase of each emitter and provide a control signal to the EOMs to stabilize the relative phase of the emitters against external thermal and acoustic noise.

The feedback bandwidth of the control system is limited to a few $\mathrm{kHz}$ by the time delay through the FPGA. To achieve $\mathrm{MHz}$ steering speeds, steering signals above the bandwidth of the control loop are applied out-of-loop by the controllers. To avoid the control loop trying to compensate for the steering signal the digital low-pass filters in the phasemeters are configured to reject the steering signal frequencies. Steering of the beam at frequencies within the bandwidth of the controller is simply achieved by sweeping the error point that the controller is trying to lock to.

The primary author can be contacted at: david.gozzard@anu.edu.au

AOS Australian Conference on Optical Fibre Technology (ACOFT) and Australian Conference on Optics, Lasers, and Spectroscopy (ACOLS) 2019, edited by Arnan Mitchell, Halina Rubinsztein-Dunlop, Proc. of SPIE Vol. 11200, 112001O - (c) 2019 SPIE · CCC code: 0277-786X/19/\$21 - doi: 10.1117/12.2539892

Proc. of SPIE Vol. $112001120010-1$ 


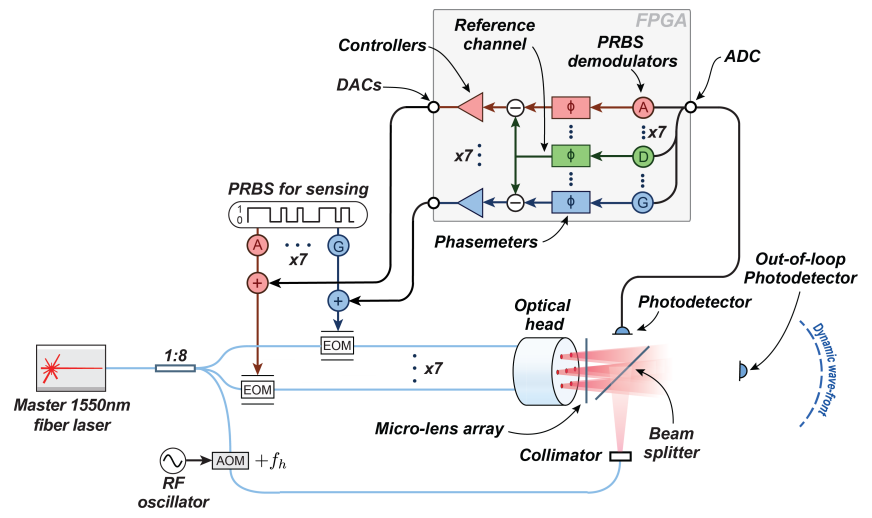

Figure 1. Simplified schematic of the experimental configuration of the OPA and digital control system. ${ }^{3}$

\section{RESULTS AND DISCUSSION}

The relative phase error between emitters governs the beam quality, the coherence of the wavefront, and the pointing precision of the OPA. The phase error was measured using an out-of-loop photodetector and separately implemented phasemeters. ${ }^{3}$ The differential RMS phase error was measured to be $25.6 \mathrm{mrad}$, corresponding to $/ 245$ cycles at $=1550 \mathrm{~nm}$, and sub-milliradian pointing precision for this array configuration.

Beam steering at frequencies of a few $\mathrm{Hz}$ could be observed directly using a beam profiler. For steering frequencies up to $1 \mathrm{MHz}$, the central lobe of the far-field interference beam will be swept across the aperture of the out-of-loop photodetector. The change in intensity as the beam transits the aperture will produce a signal at the steering frequency. The steering speed is limited to $1 \mathrm{MHz}$ by the sampling frequency of the digitalto-analogue converters (DAC) available for this experiment, but the controller architecture does not impose any inherent upper limit on the steering frequency that can be applied. Commercially available EOMs have bandwidths on the order of $40 \mathrm{GHz}$, meaning that, with a suitable high-speed DAC and associated electronics, this steering system can be scaled to $\mathrm{GHz}$ operation.

We have presented an OPA and FPGA-based digital controller architecture capable of steering a laser beam with sub-milliradian pointing precision, and progress towards demonstrating $\mathrm{MHz}$ steering rates. Higher-speed electronics and high-bandwith EOMs would make GHz steering speeds possible, and the DEHI phase measurement and feedback technique means that the system can be scaled to hundreds of individually controlled emitters given sufficient FPGA resources. OPAs based on this technology have potential applications in free-space laser communications, adaptive optics, target acquisition and tracking, satellite interferometry, and LIDAR.

\section{ACKNOWLEDGMENTS}

This work was funded by the Australian Research Council Centre of Excellence for Engineered Quantum Systems (EQUS) (project ID CE170100009), and the Australian Research Council Centre of Excellence for Gravitational Wave Discovery (OzGrav) (project ID CE170100004).

\section{REFERENCES}

[1] Roberts, L. E., Ward, R. L., Sutton, A. J., Fleddermann, R., de Vine, G., Malikides, E. A., Wuchenich, D. M., McClelland, D. E., and Shaddock, D. A., "Coherent beam combining using a 2d internally sensed optical phased array," Applied optics 53(22), 4881-4885 (2014).

[2] Shaddock, D. A., "Digitally enhanced heterodyne interferometry," Optics letters 32(22), 3355-3357 (2007).

[3] Spollard, J. T., Gozzard, D. R., Roberts, L. E., Sibley, P. G., Francis, S. P., McClelland, D. E., and Shaddock, D. A., "Towards solid-state beam steering using a 7-emitter $1550 \mathrm{~nm}$ optical phased array," in [Free-Space Laser Communications XXXI], 10910, 109101P, International Society for Optics and Photonics (2019). 